\title{
Subsidy strategy analysis of operating system vendor with network
}

\author{
externality effect \\ Xiaofeng Zhang ${ }^{a}$, Xiaojuan Zhang $^{b}$, Chunhui Cao ${ }^{c^{*}}$, Hongcai Dai ${ }^{a}$, Haixu Song ${ }^{a}$ \\ a State Grid Energy Research Institute, Beijing, China \\ b Xi'an Jiaotong University, Xi'an, China \\ c School of Management, Shanghai International Study University, Shanghai, China \\ *Corresponding author: Chunhui Cao, Assistant professor, lakerchh@163.com
}

\begin{abstract}
Owing to network externality effect is key characteristic of smartphone operating system, the vendor subsidizes app developer or consumer to extend market share. In this paper, using a two-period model framework, we study performance in cases of subsidizing developer and consumer separately. Then, the performances in two subsidy policies are compared. We find that subsidizing customer should be chosen if the development cost is low. And if the development cost is moderate, subsidizing developer can generate greater performance. Besides, the vendor must quit subsidizing if the development cost is high.
\end{abstract}

Key words: network externality effect, operating system vendor, subsidy policy, developer, consumer

\section{Introduction}

Because smartphone operating system has network externality effect, according to analysis of Suarez and Kirtley, the core of the smartphone operating system competition is increase of users $^{1}$. And users development of the smartphone operating system faces typical "first chicken or egg" dilemma. On the one hand, users refuse to use the platform because applications of which are scarce. On the other hand, application developers reject to develop applications on the platform with small user group. Therefore, it is necessary for smartphone operating system vendors to subsidize application developers and users to break the "chicken or Egg first" dilemma and quickly increase market share. Although subsidy ways of smartphone operating system vendors are varied, the effect of subsidy on application developers and users is poorly understood. And it is not possible to evaluate which subsidy strategy is more effective between application developer and user subsidy strategies.

The main discussions of the smartphone operating system focus on technical field and few discussions focus on business field. Most of the research on the business field is holistic which concentrate on the comparison of major operating systems and the prediction of the future development of the operating system. Zhije Zhang ${ }^{2}$ integrated details into holistic research which included describing profit model of the smartphone operating system based on the value chain, proposing smartphone operating system competition strategy based on 
bilateral market theory and giving smartphone operating system development strategy based on user acceptance theory. Although the existing research on the smartphone operating system is gradually detailed, the selection problem of subsidy strategy for the smartphone operating system vendors is still unsolved.

The network externality is the most basic and important feature of smartphone operating system and the key factor that can't be neglected in the operation strategy decision of smartphone operating system vendors. The network externality was first proposed by Rohlfs ${ }^{3}$ who considered that the network externality showed in positive correlation between product value and user quantity. Katz and Shapiro ${ }^{4}$ further defined the network externality as positive correlation between user utility of consuming product and user quantity. The latest researches on network externality include durable goods competition ${ }^{5}$, software pricing ${ }^{6}$, technology spillover and digital product innovation protection ${ }^{7}$, pricing in case of unequal rights between upstream and downstream of supply chain ${ }^{8}$.

Given the above, the existing researches lack exploration of subsidy strategy choice of smartphone operating system vendors based on network externality. Therefore, in this paper we study the subsidy strategy choice of the smartphone operating system vendor by comparing performance of two subsidy strategies which are provide to application developer or consumer separately.

\section{The model}

Two-stage model is considered in this paper. In the first stage, application developer decides whether to develop application. The choice of application developer is denoted by $0-1$ variable $x_{1}, x_{1}=1$ and $x_{1}=0$ represents choosing to or not to develop application. If application developer chooses not to develop in the first stage, he chooses whether to develop application again in the second stage. If application developer chooses to develop, because application has time durative, developer chooses to develop by default in the second stage. The development choice in the second stage is denoted by $0-1$ variable $x_{2}$ and $x_{1} \leq x_{2}$ according to above analysis. The application developer makes development decision as well as price of application $p_{1}$ and $p_{2}$ separately in two stages. Let the fixed development cost for application developer is $F$.

Drawing on the user's utility function in Salant ${ }^{9}$, Ellison and Fudenberg ${ }^{10}$, Zhang and Seidmann ${ }^{11}$, we set the user's utility function as $U=\theta q+e d-p$. In the user's utility function, $\theta$ indicates heterogeneity of user and normally distributed in the interval $[0,1], q_{\text {represents }}$ application quality, $e$ represents network externality strength, $d$ indicates the number of users and $p$ represents the price of application. There are three choice for users: purchasing application in the first stage, purchasing application in the second stage and no purchasing in two stages. If user chooses to purchase in the first stage, user's utility is $U_{1}=x_{1}\left(\theta q+e d_{1}-p_{1}+\delta\left(\theta q+e\left(d_{1}+d_{2}\right)\right)\right)$. If user chooses to purchase in the second stage, user's utility is $U_{2}=x_{2} \delta\left(\theta q+e\left(d_{1}+d_{2}\right)-p_{2}\right)$. If user chooses not to purchase in both stage, user's utility is 0 . In the user's utility function, $d_{1}$ and $d_{2}$ represent number of users who choose to purchase in the first and second stage respectively. Moreover, $\delta$ represents discount factor. Application developer decides whether to develop application and pricing strategy to 
maximize profit and decision function of the first stage is

$$
\max _{p_{1}, x_{1}} \pi_{1}=x_{1}\left(p_{1} d_{1}-F\right)+\delta \pi_{2}^{*}
$$

Based on decision of the first stage, decision function of second stage is

$$
\max _{p_{2}, x_{2} \geq x_{1}} \pi_{2}=\left(x_{2}-x_{1}\right)\left(p_{2} d_{2}-F\right)
$$

The sequence of events is as follows: firstly, the smartphone operating system vendor provides subsidy; secondly, in the first stage, application developer decides whether to develop according to subsidy and development cost and determines application price if the application is developed, then users choose whether to purchase application; lastly, in the second stage, application developer decides whether to develop according to decision of the first stage and development cost and determines application price if the application is developed, then users choose whether to purchase application. The order of events is shown in Figure 1.

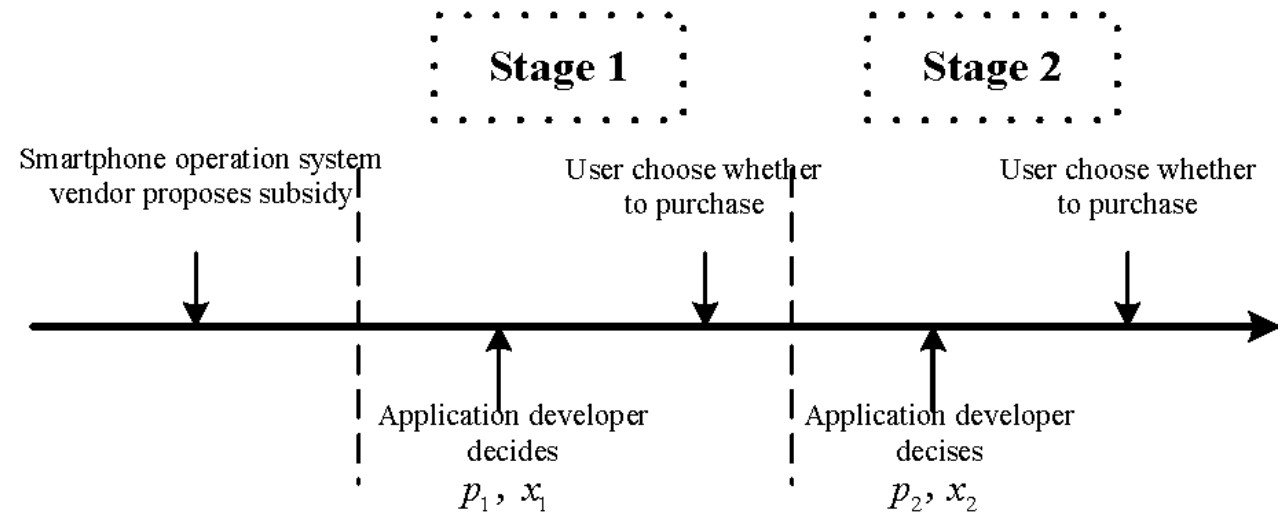

Fig.1 - Diagram of event sequence

\section{Subsidy strategy choice}

In this section, we firstly analyzes two kinds of subsidy strategies provided by smartphone operating system vendor which are application developer subsidy strategy and user subsidy strategy. Then we compare the performance of two subsidy strategies and select the subsidy strategy for the smartphone operating system vendor.

\subsection{Application developer subsidy strategy}

Suppose subsidy which is provided to application developer by smartphone operation system vender is $B^{D}$, based on subsidy strategy, decision function of application developer in the first stage changes to

$$
\max _{p_{1}, x_{1}} \pi_{1}^{D}=p_{1} d_{1}-x_{1}\left(F-B^{D}\right)+\delta \pi_{2}^{*}
$$

Decision function of application developer in the second stage changes to

$$
\max _{p_{2}, x_{2}} \pi_{2}^{D}=p_{2} d_{2}-\left(x_{2}-x_{1}\right)\left(F-B^{D}\right)
$$

By backward solution, the best decision of application developer can be obtained and is shown in Table 1. 
Table 1 - Equilibrium results of application developer subsidy

\begin{tabular}{c|c|c|c}
\hline$F$ & Price & Number of users & Profit \\
\hline \multirow{2}{*}{$F \in\left[0, F_{t}^{D}\right]$} & $p_{1}^{D}=\frac{2(1+\delta) q}{4+\delta}$ & $d_{1}^{D}=\frac{2 q}{(1-e)(4+\delta)} d_{2}^{D}=\frac{q(1+\delta)}{(1-e)(4+\delta)}$ & $\frac{q^{2}(1+\delta)}{(1-e)(4+\delta)}+B^{D}-F$ \\
& $p_{2}^{D}=\frac{q}{4+\delta}$ & $d^{D}=\frac{q(3+\delta)}{(1-e)(4+\delta)}$ & \\
\hline \multirow{3}{*}{$F \in\left(F_{t}^{D}, \infty\right)$} & $p_{1}^{D}=0$ & $d_{1}^{D}=0$ & 0 \\
& $p_{2}^{D}=0$ & $d_{2}^{D}=0$ & \\
\hline
\end{tabular}

Where $F_{t}^{D}=B^{D}+\frac{q^{2}(1+\delta)}{(1-e)(4+\delta)}$ and $d^{D}$ represents number of users in two stages that is $d^{D}=d_{1}^{D}+d_{2}^{D}$.

Proposition 1 Development decision of application developer is

(1) when development cost is in the interval $\left[0, F_{t}^{D}\right]$, application developer chooses to develop in the first stage; when development cost is in the interval $\left(F_{t}^{D}, \infty\right)$, application developer chooses not to develop.

(2) The threshold $F_{t}^{D}$ increases with network external intensity $e$ and subsidy amount $B^{D}$. According to Proposition 1, developer chooses to develop in the first stage when development cost is small. And developer abandons development when development cost is large. Moreover if development starts in the second stage, developer gets less revenue than development cost and developer will not choose to develop in the second stage. The stronger the network externality, users with the lower quality preference are attracted. Then the higher possibility that users purchase application possibility, thus development enthusiasm of developer is promoted. And developer who chooses not to develop application because of high development cost chooses to develop. And the higher the subsidy, the lower the developer's development cost and the more developers will be involved in application development.

Take WP operating system vendor as example, after proving subsidy to developer, till the third quarter of 2013, 37\% of developers develop WP applications which is far higher than $22 \%$ in August 2012. The monthly increase amount of applications in WP store is almost 800 and the number of applications exceeded 200,000 until December 2013.

\subsection{User subsidy strategy}

Subsidy that is provided to user by smartphone operating system vendor in two stage is $S_{1}^{C}$ and $S_{2}^{C}$ respectively. Based on subsidy, utility of user purchasing application in the first stage becomes $U_{1}=x_{1}\left(\theta q+e d_{1}-p_{1}+S_{1}^{C}+\delta\left(\theta q+e\left(d_{1}+d_{2}\right)\right)\right)$, utility of user purchasing application in the second stage becomes $U_{2}=x_{2} \delta\left(\theta q+e\left(d_{1}+d_{2}\right)-p_{2}+S_{2}^{C}\right)$ and utility of user not purchasing in two stages is 0 .

By backward solution, the best decision of application developer can be obtained and is 
shown in Table 2.

Table 2 - Equilibrium results of application user subsidy

\begin{tabular}{|c|c|c|c|c|}
\hline $\bar{F}$ & Price & \multicolumn{2}{|c|}{ Number of users } & Profit \\
\hline$F \in\left[0, F_{t}^{C}\right]$ & $\begin{array}{c}p_{1}^{C}=\frac{2\left((1+\delta) q+S_{1}^{C}\right)}{4+\delta} \\
p_{2}^{C}=\frac{\left(\begin{array}{l}(1+\delta)(4+\delta) S_{2}^{C} \\
+2(1+\delta) q \\
-(2+\delta) S_{1}^{C}\end{array}\right)}{2(1+\delta)(4+\delta)}\end{array}$ & $\begin{aligned} & d_{1}^{C}=\left(\begin{array}{l}(2+\delta)^{2} S_{1}^{C} \\
+4(1+\delta) q \\
-\delta(1+\delta)(4+\delta) S_{2}^{C}\end{array}\right) \\
& 2(1-e)(1+\delta)(4+\delta) \\
& d^{C}=\frac{\left(\begin{array}{l}(2+\delta) \\
+2(1+ \\
+(1+\delta\end{array}\right.}{2(1-e)(}\end{aligned}$ & $\begin{array}{l}\left(\begin{array}{l}(1+\delta)(4+\delta) S_{2}^{C} \\
+2(1+\delta) q \\
-(2+\delta) S_{1}^{C}\end{array}\right) \\
2(1-e)(4+\delta) \\
\frac{\delta) q}{(4+\delta)}\end{array}$ & $\begin{array}{l}\frac{\left(\begin{array}{l}\delta(4+\delta)(1+\delta)^{2} S_{2}^{C 2} \\
-2 \delta(1+\delta)(4+\delta) S_{1}^{C} S_{2}^{C} \\
+(2+\delta)^{2} S_{1}^{C 2} \\
+8(1+\delta) q S_{1}^{C} \\
+4(1+\delta)^{2} q^{2}\end{array}\right.}{4(1-e)(1+\delta)(4+\delta)} \\
-F\end{array}$ \\
\hline$F \in\left(F_{t}^{C}, \infty\right)$ & $\begin{array}{l}p_{1}^{C}=0 \\
p_{2}^{C}=0\end{array}$ & $\begin{array}{l}d_{1}^{C}= \\
d_{2}^{C}= \\
d^{C}=\end{array}$ & & 0 \\
\hline
\end{tabular}

Where $\quad F_{t}^{C}=\frac{\delta(1+\delta) S_{2}^{C}}{4(1-e)}-\frac{\delta S_{1}^{C} S_{2}^{C}}{2(1-e)}+\frac{(2+\delta)^{2} S_{1}^{C 2}+8(1+\delta) q S_{1}^{C}+4(1+\delta)^{2} q^{2}}{4(1-e)(1+\delta)(4+\delta)} \quad$ and $\quad d^{C} \quad$ represents number of users in two stages that is $d^{C}=d_{1}^{C}+d_{2}^{C}$.

Proposition 2 Development decision of application developer is

(1) when development cost is in the interval $\left[0, F_{t}^{C}\right]$, application developer chooses to develop in the first stage; when development cost is in the interval $\left(F_{t}^{C}, \infty\right)$, application developer chooses not to develop.

(2) The threshold $F_{t}^{C}$ increases with network external intensity $e$ and subsidy amount $S_{1}^{C}$, $S_{2}^{C}$.

Similar to Proposition 1, developer chooses to develop in the first stage when development cost is small. And developer abandons development when development cost is large. Moreover if development starts in the second stage, developer gets less revenue than development cost and developer will not choose to develop in the second stage. The stronger the network externality, users with the lower quality preference are attracted which enhance revenue of developer gained by developing application. Then developer with higher development cost chooses to develop application.

Compared with scenario without subsidy, the case that smartphone operating system vendor subsidies users will significantly expand the number of users on the platform. And the higher the subsidy, the lower the developer's development cost and the more developers will participate in application development.

\subsection{Comparison of subsidy strategies}

Integrated equilibrium results of smartphone operating system vendor providing subsidy to application developers and users separately, let subsidy amount which is provided to developer and user by smartphone operating system vendor is equal that is $B^{D}=d_{1} S_{1}^{C}+d_{2} S_{2}^{C}$. 
Then we compare performance of two subsidy strategies under equal subsidy amount and choose subsidy strategy which is better for smartphone operating system vendor. The subsidy performance is measured by the total number of users $d$.

Proposition 3 When development cost is in the interval $\left[0, F_{t}^{C}\right]$, smartphone operating system vender chooses to subsidize users; when development cost is in the interval $\left(F_{t}^{C}, F_{t}^{D}\right]$, smartphone operating system vendor chooses to subsidize developer; when development cost is in the interval $\left(F_{t}^{D}, \infty\right)$, smartphone operating system vendor chooses not to subsidize.

Intuitively, when development cost is low, subsidizing application developer by smartphone operation system vendor has very small impact on developer's decision on whether to develop application. However, in this case subsidizing users greatly affect users' decision on whether to purchase application. Therefore when development cost is low, smartphone operation system vendor prefers to subsidize users.

When development cost is relative high, subsidizing application developer by smartphone operating system vendor might change decision of application developer. In this case, enhancement performance of platform size through subsidizing application developer exceeds that through subsidizing users. Therefore, when development cost is relative high, smartphone operating system vendors prefers to subsidize application developer.

When development cost is too high, subsidy of smartphone operating system vendor is difficult to balance the developer's development cost and benefit and the incentive effect of subsidy on application developer is negligible. So that the influence of subsidy on decision-making of developer and the user can be neglected and subsidy provided by smartphone operating system vendor becomes sunk cost. Therefore when the application development cost is too high, smartphone operating system vendor chooses not to provide subsidy.

\section{Conclusions}

For a smartphone operating system vendor who is a later comer, that developers turn to develop applications based on his operating system requires relative high transfer cost. So that developers will consider developing applications based on new operating system only if there is large number of users for the operating system. For users, only when applications of new smartphone operating system is sufficient, users choose to use the operating system. The decision ways of application and users led to "First chicken with egg" dilemma. However, the core for new entrants to smartphone operating system surviving in fierce competitive market is to expand the number of platform users. Therefore, the smartphone operating system subsidize developer and user to break the user growth dilemma which are regarded as two kinds of subsidy strategies. In case of limited subsidy, it is key issue that among two subsidy strategies which one is more effective to the user growth.

In this paper, we analyze developer's decision equilibrium results in case of subsidizing application developer and user by smartphone operating system vendor. And we compare two kinds of subsidy strategies under given subsidy budget limit to find out subsidy strategy suitable for the smartphone operating system vendor. Equilibrium results under two kinds of subsidy strategies show that application developer chooses to develop in the first stage when development cost is low while application developer chooses not to develop when 
development cost is high. Comparison results of performance under two kinds of subsidy strategies show that smartphone operating system vendor prefers to subsidize user when development cost is low; smartphone operating system vendor prefers to subsidize developer when development cost is relative high and smartphone operating system vendor should abandon subsidy when development cost is too high.

In order to simplify research, in this paper we only discusses single subsidy strategy which are provided from smartphone operating system vendor to application developer and user. However, in business practice smartphone operating system vendor's subsidy strategy is more complex and diverse. For example, smartphone operating system vendor provides subsidy to both application developer and user. Smartphone operating system vendor might also subsidize brans-aware smartphone producer for producing products that carry his own system to attract users. In this paper, the above kinds of subsidy strategy haven’t been discussed and might be studies in future research.

\section{References}

1. F. F. Suarez, J. Kirtley, Dethroning an established platform, J. MIT Sloan Management Review, 53(2012) 35-41.

2. ZJ. Zhang, Research on the competition and development of the smartphone operating system, D. Beijing: Beijing University of Posts and Telecommunications, 2012.

3. J. Rohlfs, A Theory of interdependent demand for a communication service, J. The Bell Journal of Economics and Management Science, 5(1974) 16-37.

4. M. L. Katz, C. Shapiro, Network externalities, competition, and compatibility, J. The American Economic Review, 75(1985) 424-440.

5. H. Cheng, JJ. Yan, XY. Wang, A study on the competitive strategies of durable products based on network externality, J. Journal of Sichuan University (Social Science Edition), 3(2013) 62-68.

6. Y. Liu, XW. Liao, Software pricing strategy under online rating and network effect, J. Journal of Management Science, 26(2013) 60-69.

7. XD. Ma, Network externalities, technology spillovers and research on digital product innovation protection, J. Soft Science, 27(2013) 73-78.

8. H. Gong, CX. Guo, Research on pricing strategy of products with network externality under the circumstance of supply chain unfair power, J. Soft Science, 27(2013) 66-70.

9. S. W. Salant, When is inducing self-selection suboptimal for a monopolist? J. Quarterly Journal of Economics, 104(1989) 391-397.

10. G. Ellison, D. Fudenberg, The neo-luddite's lament: Excessive upgrades in the software industry, J. Rand Journal of Economics, 31(2000) 253-272.

11. J. Zhang, A. Seidmann, Perpetual versus subscription licensing under quality uncertainty and network externality effect, J. Journal of Management Information System, 27(2010) 39-68. 